\title{
Elamipretide (SS-31) Treatment Attenuates Age-Associated Post-Translational Modifications of Heart
}

\section{Proteins}

Jeremy A. Whitson ${ }^{1}$, Miguel Martín-Pérez ${ }^{2}$, Tong Zhang ${ }^{3}$, Matthew J. Gaffrey ${ }^{3}$, Gennifer E. Merrihew ${ }^{2}$,

Eric Huang ${ }^{2}$, Collin C. White ${ }^{4}$, Terrance J. Kavanagh ${ }^{4}$, Wei-Jun Qian ${ }^{3}$, Matthew D. Campbell ${ }^{5}$, Michael J. MacCoss $^{2}$, David J. Marcinek ${ }^{5}$, Judit Villén ${ }^{2}$, Peter S. Rabinovitch ${ }^{6, *}$

1. University of Washington, Department of Pathology, 1959 NE Pacific St, Seattle, WA 98195 (Current affiliation: Davidson College, Department of Biology, 405 N Main St, Davidson, NC 28035)

2. University of Washington, Department of Genome Sciences, 3720 15th Street NE, Seattle WA 98195

3. Pacific Northwest National Laboratory, Integrative Omics, 902 Battelle Boulevard, Richland, WA 99352

4. University of Washington, Department of Environmental \& Occupational Health Sciences, 4225 Roosevelt Way NE, Seattle, WA 98105

5. University of Washington, Department of Radiology, 1959 NE Pacific St, Seattle, WA 98195

6. University of Washington, Department of Pathology, 1959 NE Pacific St, Seattle, WA 98195

\section{* Corresponding Author:}

Phone: 206-685-3761; Fax: 206-616-8271

Email: petersr@u.washington.edu 


\section{ABSTRACT}

2 It has been demonstrated that elamipretide (SS-31) rescues age-related functional deficits in the 3 heart but the full set of mechanisms behind this have yet to be determined. We investigated the 4 hypothesis that elamipretide influences post-translational modifications to heart proteins. The S5 glutathionylation and phosphorylation proteomes of mouse hearts were analyzed using shotgun 6 proteomics to assess the effects of aging on these post-translational modifications and the ability of the 7 mitochondria-targeted drug elamipretide to reverse age-related changes. Aging led to an increase in 8 oxidation of protein thiols demonstrated by increased S-glutathionylation of cysteine residues on proteins 9 from Old (24 months old at the start of the study) mouse hearts compared to Young (5-6 months old). 10 This shift in the oxidation state of the proteome was almost completely reversed by 8-weeks of treatment 11 with elamipretide. Many of the significant changes that occurred were in proteins involved in mitochondrial or cardiac function. We also found changes in the mouse heart phosphoproteome that were associated with age, some of which were partially restored with elamipretide treatment. Parallel reaction monitoring of a subset of phosphorylation sites revealed that the unmodified peptide reporting for Myot S231 increased with age, but not its phosphorylated form and that both phosphorylated and unphosphorylated forms of the peptide covering CMyBP-C S307 increased, but that elamipretide treatment did not affect these changes. These results suggest that changes to thiol redox state and phosphorylation status are two ways in which age may affect mouse heart function, which can be restored by treatment with elamipretide.

KEYWORDS: Aging, mitochondria, heart, SS-31, elamipretide mass spectrometry, S-glutathionylation, phosphoproteomics, redox proteomics, post-translational modification

\section{INTRODUCTION}

Alteration of protein post-translational modifications (PTMs) is a well-established mechanism of cellular aging (Morimoto \& Cuervo, 2014; Santos \& Lindner, 2017; Walther et al., 2015). These alterations contribute to age-related dysfunction in many tissue and organ systems, including the heart which has been shown to have altered PTM profiles with age (Chiao et al., 2020). These age-associated changes could contribute to cardiac pathologies, the leading cause of death in elderly populations (Dai et al., 2012; Heron \& Anderson, 2016).

Age-related changes to PTMs include both enhancement and loss of modifications to various residues. Greater oxidative stress in the cell, primarily due to elevated mitochondrial oxidant production,

32 is a major source of PTMs that are elevated with age (Kuka et al., 2014; Santos \& Lindner, 2017). Among 
the most common oxidative modifications is reversible S-glutathionylation, including those due to the actions of glutathione-utilizing defense mechanisms that are required to prevent harmful forms of reactive oxygen species (ROS) from damaging proteins (Grek et al., 2013). S-glutathionylation can thus be viewed as a marker of general protein oxidation. We have demonstrated that mouse hearts show increased oxidative stress (Dai et al., 2012) and enhancement of S-glutathionylated residues with age (Chiao et al., 2020).

Aging also leads to dysregulation in the phosphorylation of various residues that play an important role in signaling and cellular regulation (Santos \& Lindner, 2017). We have previously shown that changes in the phosphorylation of key residues in cardiac myosin binding protein C (cMyBP-C) and cardiac troponin I (cTnl) appear to be major contributors to the age-related loss of diastolic function in the mouse heart (Chiao et al., 2020).

Restoration of proper PTM profiles has been proposed as one way to repair function in old age. Since much of this dysregulation originates from altered mitochondrial energetics and ROS, drugs that improve mitochondrial health have a strong potential to restore PTM balance. Elamipretide (also referred to in this paper by its original designation of SS-31) is a mitochondrial-targeted drug that we have previously shown to be effective at restoring function in old mouse hearts (Whitson et al., 2020). One mechanism by which elamipretide achieves this effect is by modifying PTM profiles, including enhanced CMyBP-C phosphorylation and decreased global S-glutathionylation (Chiao et al., 2020). These changes are likely secondary to its primary effect of associating with the cardiolipin-rich mitochondrial inner membrane where it improves the efficiency of the electron transport chain and other mitochondrial proteins and reduces the leak of reactive species (Campbell et al., 2019; Chavez et al., 2019; Mitchell et al., 2020; Whitson et al., 2020; Zhang et al., 2020).

To better understand the effects of elamipretide on S-glutathionylation and phosphorylation of heart protein residues, both at the level of individual proteins and across biological pathways, we used a shotgun proteomics approach to compare phosphoproteomes and thiol proteomes of Young, Old, and Old mouse hearts treated with elamipretide (Old +SS-31). While our previous work provided analysis of specific phosphorylation sites via Western blot and a measurement of bulk S-glutathionylation (Chiao et al., 2020), here we present both broader and more detailed information describing the post-translational modification of thousands of different proteins. 
Quantitative analysis of protein S-glutathionylation was performed by mass spectrometry, as previously described (Campbell et al., 2019). Aging resulted in a broad increase in S-glutathionlyation that was reversed by elamipretide (referred to as SS-31 in figures and tables) treatment (Figures $1 \mathrm{~A}$ and B). The 50 age-related S-glutathionylation changes with the lowest P-values are shown in Table 1 and the full set of results can be viewed in Appendix 1. A histogram of the S-glutathionylation occupancy measured at the peptide level illustrates an age-related increase in S-glutathionylation and shows that the elamipretide treatment in Old mice produced a general shift in S-glutathionylation almost completely back to the Young state (Figure 1C). Canonical pathway analysis by Ingeniuty Pathway Analysis (IPA) software (QIAgen) also shows that many of the significantly affected pathways are linked to mitochondria and/or aging, such as sirtuin signaling, oxidative phosphorylation, mitochondrial dysfunction, TCA cycle, and NRF2-mediated oxidative stress response (Figure 1D). Heatmap visualization of S-glutathionylation differences in peptides of selected pathways further demonstrates that elamipretide treatment strongly and uniformly shifted the oxidation state of the heart proteome back to that of Young mice (Figure 1E). Our results also show extensive S-glutathionylation of proteins involved in cardiomyocyte elasticity, such as titin, and that elamipretide greatly reduces the S-glutathionylation of these residues in aged hearts (Appendix 1; Table 1).

The global trend seen in S-glutathionylation by proteomics was further confirmed by an HPLC assay (Figure 1F). This analysis demonstrates that bulk S-glutathionylation, measured as glutathione $(\mathrm{GSH}) /$ protein after isolation and reduction, was decreased significantly $(P<0.05)$ in the heart following elamipretide treatment.

\section{Phosphoproteomics Reveals Age-Related Changes and Elamipretide Effects}

We previously described age-based changes to a small set of phosphorylation sites that are important for cardiac function (Chiao et al., 2020). To determine additional phosphosites that may regulate heart function, we analyzed the global phosphoproteome of Young, Old, and Old elamipretidetreated mouse hearts. The 50 differences between groups with the lowest P-values are shown in Table 2 and the full phosphoproteomic results can be found in Appendix 2. Many sites were identified with changes between groups of unadjusted P-value $<0.05$ (Figure 2A,B).

Canonical pathway analysis by IPA shows that elamipretide had a significant effect on many of the pathways that were altered with age and play a known role in cardiac function, such as ILK signaling, 
assessing the individual phosphosites involved these pathways, we found that the Young and Old Control hearts tended to cluster more closely together than with the Old + SS-31 hearts (Figure 2D).

To fully contextualize the phosphoproteome results, we also quantified the unmodified proteomes of the same set of samples (Figure $3 A$ and B). The 50 differentially-regulated proteins with the lowest P-values are shown in Table 3 while the full proteome results can be found in Appendix 3. Canonical pathway analysis by IPA revealed that affected pathways were largely related to cell signaling, metabolism, and structure, with limited overlap between the pathways identified in the phosphorylation data (Figure 3C). In most pathways, the Old Control and Old + SS-31 results clustered more closely together than with the Young, though the mitochondrial dysfunction pathway was a notable exception to this (Figure 3D).

The limited overlap between the significantly altered pathways in the phosphoproteomic and proteomic datasets, and the differential clustering of the groups, indicates that most of the changes found in the phosphoproteome are driven by changes in signaling and phosphoregulation of these proteins rather than differences in protein abundance.

Based on the large-scale phosphoproteomic results, we selected a subset of phosphorylation sites for further targeted analysis by parallel reaction monitoring to better assess whether they play a role in

112 the regulation of the aging heart and restoration of function by SS-31. These targets were selected from 113 sites that showed an unadjusted P-value $<0.05$ in the large-scale phosphoproteomic analysis presented 114 here, as well as sites of interest identified in our prior study (Chiao et al., 2020). These targets included phosphorylation sites on titin (Ttn), actinin alpha 2 (Acta2), cMyBP-C, myotilin (Myot), cardiac phospholamban (PIn), heat shock protein family B member 6 (HSPB6), and creatine kinase M-type (Ckm). As shown in Figure 4, we found different abundance for many phosphosites in the Young and Old groups, and some differences were significant. However, elamipretide did not appear to have any significant effects on the phosphorylation of these sites. Notably, we observed an increase in the unmodified cMyBP$C$ containing Ser307 with age $(P=0.074)$ while the corresponding phosphopeptide did not change (Figure $4 F)$. This suggests that the phosphorylation site has high stoichiometry given that there was no change in abundance when all cMyBP-C peptides were aggregated (Appendix 3). Conversely, Myot showed significant age-related increases in both phosphorylated and unmodified peptides containing the Ser231 site (Figure $4 \mathrm{G}$ and $\mathrm{H}$ ), indicating the change observed in phosphorylation is due to changes in protein expression. The full set of PRM results, including peptide sequences used, is provided in Table 4 while additional information on the sample list and peptide inclusion can be found in Appendix 4 . 


\section{Supplemental Data}

Full proteomic datasets can be found in the following appendices:

Appendix 1: S-Glutathionylation Proteomics Results

Appendix 2: DDA Phosphoproteomics Results

Appendix 3: DDA Proteomics Results

Appendix 4: PRM Phosphoproteomics Sample List and Peptide Inclusion

Raw data and Skyline document (ProteomeXchange ID: PXD024247) for PRM phosphorylation measurements is available at Panorama (https://panoramaweb.org/SS31PTM.url). Raw data for Sglutathionylation is available at MassIVE (tp://massive.ucsd.edu/MSV000085329/). Raw data for largescale DDA proteomics and phosphoproteomics have been deposited in the ProteomeXchange Consortium via the PRIDE partner repository with the dataset identifier PXD026335 (Vizcaíno et al., 2016).

Additional figures showing the results of statistical analyses are available in the Supplemental Data document.

\section{DISCUSSION}

Mitochondrial Thiols are Heavily Oxidized with Age but Elamipretide Rapidly Reverses These Changes

Our data reveal a near universal shift in heart protein thiol oxidation state with age. This is consistent with the redox stress hypothesis, which states that the loss of function that occurs with age is due to a shift in the redox state of cells to more oxidizing conditions, resulting in protein thiols becoming oxidized and disrupting signaling and other important functions of these proteins (Sohal \& Orr, 2012). Elamipretide treatment appeared to rapidly restore the environment of cardiomyocytes to a less oxidizing state and reversed the majority of the age-related S-glutathionylation after 8 weeks of treatment.

The S-glutathionylation data also appear to show an overrepresentation of mitochondrial proteins based on our pathway analysis. Given that mitochondria are the largest contributor of reactive oxygen species in the cell, it is not surprising that these pathways were heavily affected and implies potential damage to mitochondrial proteins. Although the effects of S-glutathionylation are site-specific and unknown in most cases, alteration of mitochondrial protein thiol oxidation state likely explains our previous finding that elamipretide further enhances nicotinamide mononucleotide's effects on improving mitochondrial metabolism in the aged heart when the two are administered simultaneously (Whitson et al., 2020). This could be due to changes in signaling and/or enzyme activity along mitochondrial pathways resulting from the difference in S-glutathionylation state. 
In addition to the reported correlation between phosphorylation changes and diastolic heart function (Chiao et al., 2020), there are indications that oxidative changes may also contribute to elamipretide's improvement of diastolic function in aged hearts. A recent study has revealed that cardiac stiffness is heavily influenced by the oxidation of cysteine residues along titin (Loescher et al., 2020), and many of these same residues showed enhanced S-glutathionylation with age that was reversed by elamipretide treatment in our analysis. The combined lower oxidation of essential mitochondrial and cardiac proteins may be one of the core mechanisms by which elamipretide is able to drastically improve the function of aged hearts.

Notably, these results are similar to what we had previously reported in skeletal muscle tissue, where we showed the same global shift in thiol redox status, with essential muscle and mitochondrial proteins being most affected with age and rapidly repaired by elamipretide (Campbell et al., 2019). Thus, our findings in heart can likely be extrapolated to all muscle tissue, if not to all mitochondrion-rich tissues.

While elamipretide has been clearly demonstrated to restore the redox state of aged muscle tissues to a near-young state, it has yet to be determined whether this can affect more persistent oxidative modifications of proteins beyond reversible S-glutathionylation, which may also greatly impact tissue function.

\section{How Does Elamipretide Regulate Phosphorylation?}

Phosphoproteome data were not expected to show the same global shift that the Sglutathionylation data did, given the more selective mechanisms of regulation of protein phosphorylation. We report many new sites of interest that should be further examined to gain insight into how heart protein phosphorylation changes with age and the degree of elamipretide's effect on reversing these changes. Furthermore, combining these phosphorylation results with protein abundance results demonstrates that the phosphorylation changes cannot generally be owed to a difference in protein abundance that maintains the same proportion of phosphorylation and instead represent true regulatory changes in phosphorylation status. Intriguingly, while elamipretide significantly affected phosphoregulation in many of the same pathways that aging did, the actual phosphosites impacted were often different, to the point where the phosphorylation profile of elamipretide-treated Old hearts often looked more different from Young hearts than untreated Old hearts did (Figure 2D). This seems to indicate that, while elamipretide has a restorative effect on the level of pathways and function impacted by aging, it is not always restorative at the level of individual phosphosites. Rather, there are many cases where elamipretide enhanced phosphorylation of residues that were not affected by age at all. 
The question also remains of how exactly elamipretide regulates phosphorylation given that there

192 is no direct mechanism by which it can phosphorylate or dephosphorylate proteins. The most obvious explanation for the elamipretide treatment's effect on phosphoregulation is that it results in modulation of kinase and phosphatase activity or abundance. Sites along many phosphatases and phosphatase regulators, such as PP1B, PP14C, PP2AA, and PP2BA and kinases, such as MAPK1, MAPK12, MAPK14, and PDK1, had significantly altered S-glutathionylation with age and elamipretide treatment (Appendix 1). On the level of abundance, PDK2 was the only phosphoregulator significantly affected by age, but with only a modest 1.45 -fold downregulation and no significant effect by elamipretide (Appendix 3 ). Thus, the data we have presented here suggests that elamipretide's impact on phosphorylation could be influenced by its alteration of the oxidation state of the proteome. This indicates a potential point of convergence in elamipretide's effect on both types of PTMs studied, with thiol redox proteome changes playing a role in regulating the phosphoproteome changes. Confirming which kinases and phosphatases play a role in aging and elamipretide's effects, and whether they are sensitive to oxidative modifications, could be beneficial to further understand cardiac aging and the mechanisms of elamipretide and should be a focus of future research.

\section{Conclusions} states of cardiac proteins and indicate that elamipretide has a potent effect on the thiol redox status of essential mitochondrial and muscle proteins while also influencing the phosphorylation of various proteins in cardiomyocytes. With these data we postulate phosphatases could be a potentially critical

212 mediator of elamipretide's effects, a possibility that needs further investigation. In total, the data we have 213 presented here includes many new targets for study in the restoration of cardiac function in old age and 214 has contributed to defining how elamipretide confers its benefits to cardiac healthspan.

\section{EXPERIMENTAL PROCEDURES}

\section{Animal Use and Care}

All mice used in this study were males of the C57BL/6 strain. Young and Old mice were obtained from the National Institute on Aging Charles River colony and further aged to 5-6 and 24 months,

221 accredited facility under Institutional Animal Care and Use Committee supervision with ad-libitum access

222 to food and water. Old mice were randomly assigned to Control and elamipretide (Old + SS-31) groups. 


\section{Drug Administration and Treatment Groups}

Elamipretide was provided by Stealth BioTherapeutics (Newton, MA) and administered at a 3 $\mathrm{mg} / \mathrm{kg}$ body weight/day dosage through osmotic minipumps (ALZET, Cupertino, CA) implanted surgically under the skin on the left dorsal side of the mice. Old Control mice were implanted with saline-containing pumps. After 4 weeks, the original minipump was surgically removed and a new minipump was implanted to continue the treatment for another 4 weeks.

\section{Euthanasia and Tissue Handling}

Mice were euthanized by live cervical dislocation. Hearts were immediately removed, rinsed with PBS, and weighed. Tissue was cut into $\sim 2 \mathrm{~mm}^{3}$ chunks and snap frozen in liquid $\mathrm{N}_{2}$ to store for further processing. Frozen tissue was mechanically lysed into a fine powder using a Tissuelyser II (QIAgen, Hilden, Germany) prior to mass spectroscopy-based procedures, with the exception of protein S236 glutathionylation.

\section{Large-Scale DDA Abundance and Phosphorylation Analysis} Sample preparation for proteomic analysis

About $50 \mathrm{mg}$ of ground tissue were resuspended in $1600 \mu \mathrm{L}$ of lysis buffer composed of $8 \mathrm{M}$ urea, $75 \mathrm{mM} \mathrm{NaCl}, 50 \mathrm{mM}$ Tris $\mathrm{pH} \mathrm{8.2,} \mathrm{and} \mathrm{a} \mathrm{mix} \mathrm{of} \mathrm{protease} \mathrm{inhibitors} \mathrm{(Roche} \mathrm{Complete} \mathrm{EDTA-free)} \mathrm{and}$ phosphatase inhibitors (50 mM beta-glycerophosphate, $10 \mathrm{mM}$ sodium pyrophosphate, $1 \mathrm{mM}$ sodium orthovanadate and $50 \mathrm{mM}$ sodium fluoride). Samples were then subjected to 2 cycles of bead beating ( 1 min beating, $1.5 \mathrm{~min}$ rest) with $0.5 \mathrm{~mm}$ diameter zirconia beads and sonicated for $5 \mathrm{~min}$ in ice. Samples were centrifuged at $4{ }^{\circ} \mathrm{C}$ to remove debris and lysate protein concentration was measured by BCA assay

246 (Thermo Fisher Scientific, Waltham, MA). Protein was reduced with $5 \mathrm{mM}$ dithiothreitol (DTT) for 30 min at 55ㅇ $\mathrm{C}$ and alkylated with $15 \mathrm{mM}$ iodoacetamide in the dark for $30 \mathrm{~min}$ at room temperature. The alkylation reaction was quenched by incubating with additional $5 \mathrm{mM}$ DTT for $15 \mathrm{~min}$ at room temperature. Samples were diluted five-fold with $50 \mathrm{mM}$ Tris $\mathrm{pH}$ 8.2. Proteolytic digestion was performed by adding trypsin at 1:200 enzyme to protein ratio and incubating at 37으 overnight. The digestion was quenched by addition of trifluoroacetic acid to $\mathrm{pH} 2$. Samples were centrifuged to remove insoluble material and peptides were desalted over a $50 \mathrm{mg}$ tC18 SepPak cartridge (Waters Corp, Milford, MA). 
of $0.1 \%$ trifluoroacetic acid. Then peptide samples were loaded into the cartridges, washed with $3 \mathrm{~mL}$ of $0.1 \%$ trifluoroacetic acid and $1 \mathrm{~mL}$ of $0.5 \%$ acetic acid, and then sequentially eluted first with $0.5 \mathrm{~mL}$ of $40 \%$ acetonitrile, $0.5 \%$ acetic acid and then with $0.5 \mathrm{~mL}$ of $70 \%$ acetonitrile, $0.25 \%$ acetic acid. $20 \mu \mathrm{g}$ and $500 \mu \mathrm{g}$ aliquots of eluted peptides were dried by vacuum centrifugation and stored at -80 으 for proteomic and phosphoproteomic analysis, respectively.

Phosphopeptide enrichment

Phosphopeptide enrichment was done by immobilized metal affinity chromatography (IMAC). 500 $\mu \mathrm{g}$ of peptides were resuspended in $150 \mu \mathrm{l} 80 \%$ acetonitrile, $0.1 \%$ trifluoroacetic acid. To prepare IMAC slurry, Ni-NTA magnetic agarose (Qiagen) was stripped with 40 mM EDTA for 30 min, reloaded with 10 $\mathrm{mM} \mathrm{FeCl} 3$ for $30 \mathrm{~min}$, washed 3 times and resuspended in $80 \%$ acetonitrile, $0.1 \%$ trifluoroacetic acid. Phosphopeptide enrichment was performed using a KingFisher Flex robot (Thermo Fisher Scientific) programmed to incubate peptides with $150 \mu \mathrm{l}$ 5\% bead slurry for $30 \mathrm{~min}$, wash 3 times with $150 \mu \mathrm{l} 80 \%$ acetonitrile, $0.1 \%$ trifluoroacetic acid, and elute with $60 \mu \mathrm{l} 1: 1$ acetonitrile:1\% ammonium hydroxide. The eluates were acidified with $30 \mu \mathrm{l}$ 10\% formic acid, $75 \%$ acetonitrile, dried by vacuum centrifugation, and stored at $-80^{\circ} \mathrm{C}$ until mass spectrometry analysis.

\section{LC-MS/MS analysis}

Peptide and phosphopeptide samples were dissolved in 4\% formic acid, 3\% acetonitrile, loaded onto a $100 \mu \mathrm{m}$ ID x $3 \mathrm{~cm}$ precolumn packed with Reprosil C18 $3 \mu \mathrm{m}$ beads (Dr. Maisch GmbH), and separated by reverse phase chromatography on a $100 \mu \mathrm{m}$ ID x $30 \mathrm{~cm}$ analytical column packed with 1.9 $\mu \mathrm{m}$ beads of the same material and housed into a column heater set at $50^{\circ} \mathrm{C}$. As peptides eluted off the column, they were analyzed online by mass spectrometry. Peptides for proteome analysis were eluted into a Q-Exactive (Thermo Fisher Scientific) mass spectrometer by gradient elution delivered by an Easyll nanoLC system (Thermo Fisher Scientific). The gradient was $9-30 \%$ acetonitrile in $0.125 \%$ formic acid over the course of $90 \mathrm{~min}$. The total duration of the method, including column wash and equilibration was 120 min. All MS spectra were acquired on the orbitrap mass analyzer and stored in centroid mode. Full MS scans were acquired from 300 to $1500 \mathrm{~m} / \mathrm{z}$ at 70,000 FWHM resolution with a fill target of $3 \mathrm{E} 6$ ions and maximum injection time of $100 \mathrm{~ms}$. The 20 most abundant ions on the full MS scan were selected for fragmentation using $2 \mathrm{~m} / \mathrm{z}$ precursor isolation window and beam-type collisional-activation dissociation (HCD) with $26 \%$ normalized collision energy. MS/MS spectra were collected at 17,500 FWHM resolution with a fill target of 5E4 ions and maximum injection time of $50 \mathrm{~ms}$. Fragmented precursors were dynamically excluded from selection for $30 \mathrm{~s}$. Phosphopeptides for phosphoproteome analysis were eluted into a Velos Orbitrap (Thermo Fisher Scientific) mass spectrometer by gradient elution delivered 
by an Easy1000 nanoLC system (Thermo Fisher Scientific). The gradient was 9-23\% acetonitrile in 0.125\% formic acid over the course of $90 \mathrm{~min}$. The total duration of the method, including column wash and equilibration was 120 min. Full MS scans were acquired in the orbitrap mass analyzer and recorded in centroid mode. Mass range was 300 to 1500 , resolution 60,000 FWHM, fill target 3E6 ions, and maximum injection time $100 \mathrm{~ms}$. Each MS scan was followed by up to 20 data-dependent MS/MS scans on the top 20 most intense precursor ions with $2 \mathrm{~m} / \mathrm{z}$ isolation window, collision-induced dissociation (CID) with 35\% normalized collision energy and acquired on the ion trap. Fragmented precursors were dynamically excluded from selection for $30 \mathrm{~s}$. MS data analysis

Acquired mass spectra raw files were converted to mzXML format and MS/MS spectra were searched against the mouse SwissProt database including isoforms (downloaded May 10, 2015, 24,750 protein sequences) using the Comet search algorithm (version 2015.02 rev.2) (Eng et al., 2013). Search parameters included full tryptic enzyme specificity with up to two missed cleavages permitted, mass tolerance of $50 \mathrm{ppm}$ for the precursor and $1 \mathrm{Da}$ for fragments ions, fixed modifications of carbamidomethylation on cysteines, and as variable modifications methionine oxidation and protein $\mathrm{N}$ terminal acetylation. Phosphorylation on serine, threonine and tyrosine residues was also included as variable modification in phosphoproteome analysis. Peptide matches were filtered to $<1 \%$ false-discovery rate, using the target-decoy database strategy and Percolator (version 3.1.2) (Käll et al., 2007). Protein inference was carried out using Protein Prophet (Nesvizhskii et al., 2003) and protein groups were filtered at $\geq 90 \%$ probability score. Peptides were quantified using in-house software by peak-area integration of MS1 spectra, peptide intensities were added for every protein group for protein intensity measurements whereas phosphopeptide intensities were treated individually. Phosphorylation site localization was performed using an in-house implementation of Ascore (Beausoleil et al., 2006) and sites with an Ascore

311 If Ascore $<13$ the most likely position is indicated including into brackets the range of residues towards the

$312 \mathrm{~N}$ and $\mathrm{C}$ termini of the phosphopeptide where other phospho-acceptor sites reside. Perseus software

313 (Tyanova et al., 2016) was used for bioinformatic and statistical analysis using log2 transformed data from 314 total intensity normalized protein intensities and median normalized phosphopeptide intensities from 315 each run. 
Powdered heart tissue was solubilized in $50 \mathrm{mM}$ triethylammonium bicarbonate (TEAB) pH 7.55 buffer with 5\% SDS, $2 \mathrm{mM} \mathrm{MgCl}_{2}$, and HALT phosphatase and protease inhibitors (Thermo Fisher Scientific). Eight-hundred ng of enolase were added to each $50 \mu \mathrm{g}$ sample for normalization. Lysates were bound to S-Trap mini columns, washed with TEAB-buffered methanol and a 1:1 mix of chloroform and methanol, digested with trypsin, and eluted following the manufacturer's protocol (Profiti, Farmingdale, NY). Eluents were dried using a CentriVap Concentrator (LABCONCO, Kansas City, MO) and reconstituted in $0.1 \%$ formic acid.

One ug of each sample with 50 femtomole of heavy labeled Peptide Retention Time Calibrant (PRTC) mixture (Thermo Fisher Scientific, cat \# 88321) was loaded onto a $30 \mathrm{~cm}$ fused silica picofrit (New Objective, Littleton, MA) $75 \mu \mathrm{m}$ column and $4 \mathrm{~cm} 150 \mu \mathrm{m}$ fused silica Kasil1 (PQ Corporation, Malvern, PA) frit trap loaded with $3 \mu \mathrm{m}$ Reprosil-Pur C18 (Dr. Maisch) reverse-phase resin analyzed with a Thermo Easy-nLC 1200. The PRTC mixture is used to assess system suitability (QC) before and during analysis. Buffer $A$ was $0.1 \%$ formic acid in water and buffer B was $0.1 \%$ formic acid in $80 \%$ acetonitrile. The 40 minute system suitability gradient consisted of a 0 to $16 \%$ B in 5 minutes, 16 to $35 \%$ B in 20 minutes, 35 to $75 \%$ B in 1 minute, 75 to $100 \%$ B in 5 minutes, followed by a wash of 9 minutes and a 30 -minute column equilibration. The 110-minute sample LC gradient consists of a 2 to $7 \%$ for 1 minutes, 7 to $14 \%$ B in 35 minutes, 14 to $40 \%$ B in 55 minutes, 40 to $60 \%$ B in 5 minutes, 60 to $98 \%$ B in 5 minutes, followed by a 9 minute wash and a 30-minute column equilibration. Peptides were eluted from the column with a $50^{\circ} \mathrm{C}$ heated source (CorSolutions, Ithica, NY) and electrosprayed into a Thermo Orbitrap Fusion Lumos Mass Spectrometer with the application of a distal $3 \mathrm{kV}$ spray voltage. For the sample digest, a full-scan mass spectrum at 60,000 resolution with a mass range of 400 to $2000 \mathrm{~m} / \mathrm{z}$, AGC target of 4e5, 50 ms maximum injection time was followed by 81 unscheduled PRM scans at 15,000 resolution with a mass range of 150 to $2000 \mathrm{~m} / \mathrm{z}$, AGC target of 5e5, $22 \mathrm{~ms}$ maximum injection time and 27\% NCE. Application of the mass spectrometer and LC solvent gradients are controlled by the ThermoFisher XCalibur (version 3.3.2782.34) data system.

Thermo RAW files were converted to mzML format using Proteowizard (version 3.0.19113) and 344 imported into a Skyline document (daily version 20.1.9.234) configured with inclusion peptides (Appendix 345 3). A DDA with MS1 filtering search was performed using MSAmanda (with built-in Percolator) in Skyline 346 filtering for PRM peptides with a default cut-off of 0.95. Parameters used included a fixed 347 carbamidomethyl modification of 57 Da on Cysteine, up to 3 variable phosphorylation modifications of 80 348 Da on Serine and Threonine, MS1 settings of precursor charge state of 2 and a mass accuracy of $10 \mathrm{ppm}$ 349 with centroided peaks. MS2 settings were fully tryptic allowing for 2 missed cleavages, fragment b- and y- 
ions, retention time filtering of scans within 5 minutes of MS2 IDs at a tolerance of $10 \mathrm{ppm}$ using the Uniprot mouse canonical FASTA. MSAmanda mzid output files were imported with default cut-off of 0.95 to build a PRM spectral library. Data was then normalized to TIC and mean normalized areas of peptides were exported from Skyline. Due to differences in detection of peptides, only the first batch of samples was able to be quantified for the desired set of peptides. Only peptides that were detected in both the phosphorylated and unphosphorylated state are presented in this paper.

\section{Thiol Redox Proteomics}

A tandem mass tag (TMT)-based quantitative redox proteomics approach was used to measure the relative protein-SSG modification levels in Young, Old Control, and Old + SS-31 heart tissue exactly as described previously for skeletal muscle tissue (Kramer et al., 2018).

\section{Protein S-Glutathionylation HPLC Analysis}

Previously snap-frozen heart tissue was homogenized in $1 \mathrm{ml} \mathrm{pH} 8$ HEPES buffer containing 75 $\mathrm{mM}$ monobromobimane (MBB). The sample was incubated for $30 \mathrm{~min}$ at room temperature to label free thiols with MBB, and then acidified with $10 \%$ sulfosalicylic acid (SSA) to precipitate proteins and stabilize GSH-bimane free conjugate. The free thiol supernatant was then removed and the protein pellet was washed $2 \mathrm{X}$ with an aliquot of $10 \%$ SSA followed by re-solubilization of the protein for $30 \mathrm{~min}$ at $60{ }^{\circ} \mathrm{C}$ in $0.5 \mathrm{~N} \mathrm{NaOH}$. After re-solubilization of the protein pellet the sample was brought to $\mathrm{pH} 7$ with $0.1 \mathrm{~N} \mathrm{HCL}$, and the protein-bound glutathione was released by a 30-min incubation in $10 \mathrm{mM}$ tris(2carboxyethyl)phosphine (TCEP). The solution was then incubated with $1 \mathrm{mM} \mathrm{MBB}$ for 30-minutes. The GSH-bimane conjugate was stabilized by addition of $10 \% \mathrm{SSA}$ to $\mathrm{pH} 2$, and the sample was then centrifuged to pellet precipitated proteins. A 50 ul aliquot of the supernatant was then injected into a Shimadzu HPLC for quantitation of the GSH-bimane conjugate as previously reported (White et al., 1999), using total protein levels from split samples as the denominator (Bradford protein assay, Bio-Rad, Hercules, CA).

\section{Statistical Analysis}

Statistical analyses of large-scale proteomics data are described in the above sections. Canonical pathway analysis was performed using Ingenuity Pathway Analysis (IPA) software (QIAgen). PRM results, were analyzed by one-way ANOVA as appropriate using Prism software (GraphPad Software, San Diego, CA). All bar charts are plotted as means \pm SEM. 


\section{ACKNOWLEDGEMENTS}

Funding for this research was provided by the UW Genetic Approaches to Aging Training Grant (T32AG000057-40), NIH/NIA grant P01AG001751, NIH/NIEHS grant P30ES007033, and the UW Nathan Shock Center. The thiol redox proteomics experiments described herein were performed in the Environmental Molecular Sciences Laboratory, Pacific Northwest National Laboratory, a national scientific user facility sponsored by the Department of Energy under Contract DE-AC05-76RL0 1830. SS31 was provided by Stealth Therapeutics (Newton, MA) free of charge. Stealth Therapeutics did not play any role in the experimental design, data collection, or authorship of this research.

\section{REFERENCES}

Beausoleil, S. A., Villén, J., Gerber, S. A., Rush, J., \& Gygi, S. P. (2006). A probability-based approach for high-throughput protein phosphorylation analysis and site localization. Nature Biotechnology, 24(10), 1285-1292. https://doi.org/10.1038/nbt1240

Campbell, M. D., Duan, J., Samuelson, A. T., Gaffrey, M. J., Merrihew, G. E., Egertson, J. D., Wang, L., Bammler, T. K., Moore, R. J., White, C. C., Kavanagh, T. J., Voss, J. G., Szeto, H. H., Rabinovitch, P. S., MacCoss, M. J., Qian, W. J., \& Marcinek, D. J. (2019). Improving mitochondrial function with SS-31 reverses age-related redox stress and improves exercise tolerance in aged mice. Free Radical Biology and Medicine, 134, 268-281. https://doi.org/10.1016/j.freeradbiomed.2018.12.031

Chavez, J. D., Tang, X., Campbell, M. D., Reyes, G., Kramer, P. A., Stuppard, R., Keller, A., Marcinek, D. J., \& Bruce, J. E. (2019). Mitochondrial protein interaction landscape of SS-31. BioRxiv, 739128. https://doi.org/10.1101/739128

Chiao, Y. A., Zhang, H., Sweetwyne, M., Whitson, J., Ting, Y. S., Basisty, N., Pino, L. K., Quarles, E., Nguyen, N. H., Campbell, M. D., Zhang, T., Gaffrey, M. J., Merrihew, G., Wang, L., Yue, Y., Duan, D., Granzier, H. L., Szeto, H. H., Qian, W. J., ... Rabinovitch, P. (2020). Late-life restoration of mitochondrial function reverses cardiac dysfunction in old mice. ELife, 9, 1-26. https://doi.org/10.7554/eLife.55513

Dai, D.-F., Chen, T., Johnson, S. C., Szeto, H., \& Rabinovitch, P. S. (2012). Cardiac aging: from molecular mechanisms to significance in human health and disease. Antioxidants \& Redox Signaling, 16(12), 1492-1526. https://doi.org/10.1089/ars.2011.4179

Eng, J. K., Jahan, T. A., \& Hoopmann, M. R. (2013). Comet: An open-source MS/MS sequence database 
search tool. Proteomics, 13(1), 22-24. https://doi.org/10.1002/pmic.201200439

413

414

415

416

417

418

419

420

421

422

423

424

425

426

427

428

429

430

431

432

433

434

435

436

437

438

439

Grek, C. L., Zhang, J., Manevich, Y., Townsend, D. M., \& Tew, K. D. (2013). Causes and consequences of cysteine s-glutathionylation. In Journal of Biological Chemistry (Vol. 288, Issue 37, pp. 2649726504). American Society for Biochemistry and Molecular Biology. https://doi.org/10.1074/jbc.R113.461368

Heron, M., \& Anderson, R. N. (2016). Changes in the Leading Cause of Death: Recent Patterns in Heart Disease and Cancer Mortality. NCHS Data Brief, 254, 1-8.

Käll, L., Canterbury, J. D., Weston, J., Noble, W. S., \& MacCoss, M. J. (2007). Semi-supervised learning for peptide identification from shotgun proteomics datasets. Nature Methods, 4(11), 923-925. https://doi.org/10.1038/nmeth1113

Kramer, P. A., Duan, J., Gaffrey, M. J., Shukla, A. K., Wang, L., Bammler, T. K., Qian, W. J., \& Marcinek, D. J. (2018). Fatiguing contractions increase protein S-glutathionylation occupancy in mouse skeletal muscle. Redox Biology, 17, 367-376. https://doi.org/10.1016/j.redox.2018.05.011

Kuka, S., Tatarkova, Z., Racay, P., Lehotsky, J., Dobrota, D., \& Kaplan, P. (2014). Effect of aging on formation of reactive oxygen species by mitochondria of rat heart. General Physiology and Biophysics, 32(03), 415-420. https://doi.org/10.4149/gpb_2013049

Loescher, C. M., Breitkreuz, M., Li, Y., Nickel, A., Unger, A., Dietl, A., Schmidt, A., Mohamed, B. A., Kötter, S., Schmitt, J. P., Krüger, M., Krüger, M., Toischer, K., Maack, C., Leichert, L. I., Hamdani, N., \& Linke, W. A. (2020). Regulation of titin-based cardiac stiffness by unfolded domain oxidation (UnDOx). Proceedings of the National Academy of Sciences of the United States of America, 117(39), 2454524556. https://doi.org/10.1073/pnas.2004900117

Mitchell, W., Ng, E. A., Tamucci, J. D., Boyd, K. J., Sathappa, M., Coscia, A., Pan, M., Han, X., Eddy, N. A., May, E. R., Szeto, H. H., \& Alder, N. N. (2020). The mitochondria-targeted peptide SS-31 binds lipid bilayers and modulates surface electrostatics as a key component of its mechanism of action. Journal of Biological Chemistry, 295(21), 7452-7469. https://doi.org/10.1074/jbc.RA119.012094

Morimoto, R. I., \& Cuervo, A. M. (2014). Proteostasis and the aging proteome in health and disease. The Journals of Gerontology. Series A, Biological Sciences and Medical Sciences, 69 Suppl 1, S33-8. https://doi.org/10.1093/gerona/glu049 
440

Nesvizhskii, A. I., Keller, A., Kolker, E., \& Aebersold, R. (2003). A statistical model for identifying proteins by tandem mass spectrometry. Analytical Chemistry, 75(17), 4646-4658. https://doi.org/10.1021/ac0341261

Santos, A. L., \& Lindner, A. B. (2017). Protein Posttranslational Modifications: Roles in Aging and AgeRelated Disease. Oxidative Medicine and Cellular Longevity, 2017, 5716409. https://doi.org/10.1155/2017/5716409

Sohal, R. S., \& Orr, W. C. (2012). The redox stress hypothesis of aging. In Free Radical Biology and Medicine (Vol. 52, Issue 3, pp. 539-555). Free Radic Biol Med. https://doi.org/10.1016/j.freeradbiomed.2011.10.445

Tyanova, S., Temu, T., Sinitcyn, P., Carlson, A., Hein, M. Y., Geiger, T., Mann, M., \& Cox, J. (2016). The Perseus computational platform for comprehensive analysis of (prote)omics data. In Nature Methods (Vol. 13, Issue 9, pp. 731-740). Nature Publishing Group. https://doi.org/10.1038/nmeth.3901

Vizcaíno, J. A., Csordas, A., Del-Toro, N., Dianes, J. A., Griss, J., Lavidas, I., Mayer, G., Perez-Riverol, Y., Reisinger, F., Ternent, T., Xu, Q. W., Wang, R., \& Hermjakob, H. (2016). 2016 update of the PRIDE database and its related tools. Nucleic Acids Research, 44(D1), D447-D456. https://doi.org/10.1093/nar/gkv1145

Walther, D. M., Kasturi, P., Mann, M., Ulrich, F., \& Correspondence, H. (2015). Widespread Proteome Remodeling and Aggregation in Aging C.\&nbsp;elegans. https://doi.org/10.1016/j.cell.2015.03.032

White, C. C., Krejsa, C. J., Eaton, D. L., \& Kavanagh, T. J. (1999). HPLC-Based Assays for Enzymes of Glutathione Biosynthesis. Current Protocols in Toxicology, 00(1), 6.5.1-6.5.14. https://doi.org/10.1002/0471140856.tx0605s00

Whitson, J. A., Bitto, A., Zhang, H., Sweetwyne, M. T., Coig, R., Bhayana, S., Shankland, E. G., Wang, L., Bammler, T. K., Mills, K. F., Imai, S., Conley, K. E., Marcinek, D. J., \& Rabinovitch, P. S. (2020). SS-31 and NMN: Two paths to improve metabolism and function in aged hearts. Aging Cell. https://doi.org/10.1111/acel.13213

Zhang, H., Alder, N. N., Wang, W., Szeto, H., Marcinek, D. J., \& Rabinovitch, P. S. (2020). Reduction of elevated proton leak rejuvenates mitochondria in the aged cardiomyocyte. ELife, 9, 1-18. https://doi.org/10.7554/ELIFE.60827 
470 SUPPORTING INFORMATION

471 TABLES

472 Table 1. Top 50 age-related S-glutathionylation changes in mouse hearts. Bold numbers indicate

473 significance (FDR<0.1). Ordered by lowest P-value in Young vs Old Control comparison. Full results can be

474 found in Appendix 1.

\begin{tabular}{|c|c|c|c|}
\hline \multirow[t]{2}{*}{ Peptide } & \multirow{2}{*}{$\begin{array}{l}\text { Maps to } \\
\text { Protein }\end{array}$} & \multicolumn{2}{|c|}{$\begin{array}{l}\text { Fold Change Relative } \\
\text { to Old Control }\end{array}$} \\
\hline & & Young & Old SS-31 \\
\hline K.VCIVGSGNWGSAIAK.I & Gpd1 & -1.42 & -1.20 \\
\hline R.SNTGGQAFPQCVFDHWQILPGDPFDNSSRPSQVVAETR.K & Eef2 & -1.69 & -1.18 \\
\hline R.YADLTEDQLPSCESLKDTIAR.A & Pgam1 & -1.45 & -1.22 \\
\hline R.GFLTERDDILCPDCGKDI.- & $F h / 2$ & -1.51 & -1.35 \\
\hline K.AEFASACTPGLHVDLSAAGSEIKPEVSSSMPAAVDVLR.T & Perm1 & -1.36 & -1.23 \\
\hline R.WGLGGTCVNVGCIPK.K & Txnrd1 & -1.39 & -1.19 \\
\hline K.CEFDPLHTVLLK.D & Cobll1 & -1.47 & -1.33 \\
\hline R.LDGNPLTQSSLPPDMYECLR.V & Lum & -1.46 & -1.25 \\
\hline R.HLLPLVQCPTLIVHGEKDPLVPR.F & Bphl & -1.49 & -1.41 \\
\hline R.AGKPVICATQM*LESMIK.K & $P k m$ & -1.53 & -1.28 \\
\hline K.SSFATPGVNVGLFCSTPAVALGR.A & Echdc3 & -1.56 & -1.28 \\
\hline K.CNENYTTDFIFNLYSEEGK.G & Pfkm & -1.61 & -1.39 \\
\hline K.GLIAAICAGPTALLAH.E & Park7 & -2.01 & -1.77 \\
\hline R.KTETVCTFQDGALVQHQQWDGK.E & Fabp5 & -1.57 & -1.34 \\
\hline R.EGVM*EFLLANHPLDC\#PICDQGGEC\#DLQDQSMMFGSDR.S & Ndufs1 & -2.30 & -1.53 \\
\hline K.VIDVTVPLQCLVK.D & Dnajc11 & -1.49 & -1.32 \\
\hline R.PFDIRTECFVPDDKEEYVK.A & Myh6 & -1.32 & -1.27 \\
\hline K.EKPYFPIPEDCTFIQNVPLEDR.V & Hnrnpu & -1.52 & -1.29 \\
\hline K.SSAAQAIHPGYGFLSENMEFAELCK.Q & Mccc1 & -1.46 & -1.27 \\
\hline K.LCYVALDFENEM*ATAASSSSLEK.S & Acta2 & -1.58 & -1.37 \\
\hline R.SSLWPMTFGLAC\#CAVEMMHMAAPR.Y & Ndufs7 & -4.07 & -2.30 \\
\hline K.YGALVICETPEQIANLEEVGR.R & Tsfm & -1.57 & -1.29 \\
\hline K.KWDTCAPEVILHAVGGK.L & Bpnt1 & -1.69 & -1.48 \\
\hline R.QELQVIADLCVK.H & Kyat3 & -1.40 & -1.25 \\
\hline K.SLCPETWPMWAGRPQDGVAVLVR.H & Myo1c & -1.46 & -1.35 \\
\hline R.SAVQYAECQSK.A & Manba & -1.21 & -1.17 \\
\hline R.IDVAVNCAGIAVAIK.T & $H s d 17 b 10$ & -1.95 & -1.52 \\
\hline K.ELLFYCSLPQSR.E & Snta1 & -1.45 & -1.36 \\
\hline K.DIRVCLVEK.A & Etfdh & -1.65 & -1.42 \\
\hline R.SCQTALAEILDVLVR.A & lars2 & -1.61 & -1.45 \\
\hline K.LCYVALDFEQEM*ATAASSSSLEK.S & Actb & -1.69 & -1.41 \\
\hline K.TVVNISSLCALQPYK.G & Spr & -1.54 & -1.32 \\
\hline K.STQFEYAWCLVR.S & Fis1 & -1.44 & -1.28 \\
\hline K.CAELEEELK.T & Tpm1 & -1.66 & -1.22 \\
\hline
\end{tabular}




\begin{tabular}{|l|c|c|c|}
\hline K.VGKDELFALEQSCAQVVLQAANER.N & Fscn1 & $\mathbf{- 2 . 3 7}$ & $\mathbf{- 1 . 7 6}$ \\
\hline K.MLTEAIMHDCVVK.L & Eif4g1 & $\mathbf{- 1 . 5 0}$ & $\mathbf{- 1 . 3 2}$ \\
\hline K.KLNCQVIGASVDSHFCHLAWINTPK.K & Prdx1 & $\mathbf{- 1 . 4 0}$ & $\mathbf{- 1 . 2 6}$ \\
\hline K.ALAGCDFLTISPK.L & Taldo1 & $\mathbf{- 1 . 7 6}$ & -1.38 \\
\hline K.LTHAAPCMLFMK.G & Glrx3 & $\mathbf{- 1 . 3 3}$ & $\mathbf{- 1 . 2 1}$ \\
\hline K.SGSTVFAEIQGVIDACIK.L & Ap3m1 & $\mathbf{- 2 . 1 0}$ & $\mathbf{- 1 . 7 7}$ \\
\hline K.NDPPMEAAGFTAQVIILNHPGQISAGYAPVLDCHTAHIACK.F & Eef1a1 & $\mathbf{- 1 . 4 8}$ & $\mathbf{- 1 . 2 9}$ \\
\hline R.CNSVLPGFIATPMTQK.M & Hsd17b8 & $\mathbf{- 1 . 3 7}$ & $\mathbf{- 1 . 2 1}$ \\
\hline K.CPKPVIAAIHGGCIGGGVDLVSACDIR.Y & Ech1 & $\mathbf{- 1 . 4 2}$ & $\mathbf{- 1 . 2 7}$ \\
\hline R.NAGIEAQVWKPSFCFK.- & Synpo & $\mathbf{- 1 . 9 1}$ & $\mathbf{- 1 . 5 4}$ \\
\hline K.ISNVGEDSCTVQWEPPAYDGGQPVLGYILER.K & cMyBP-C & $\mathbf{- 1 . 4 6}$ & $\mathbf{- 1 . 3 1}$ \\
\hline K.TGQATVASGIPAGWMGLDCGTESSKK.Y & Pgk1 & $\mathbf{- 1 . 3 7}$ & $\mathbf{- 1 . 1 8}$ \\
\hline K.TYILTCEHDVLRDDGIMYAK.R & Nceh1 & $\mathbf{- 2 . 4 0}$ & $\mathbf{- 1 . 6 7}$ \\
\hline R.ILEGMGVTFHCK.M & Ttn & $\mathbf{- 1 . 4 8}$ & $\mathbf{- 1 . 3 2}$ \\
\hline K.ILDVGCGGGLLTEPLGR.L & Coq3 & $\mathbf{- 1 . 5 5}$ & $\mathbf{- 1 . 2 8}$ \\
\hline R.KGLIAAICAGPTALLAHEVGFGCK.V & Park7 & $\mathbf{- 2 . 1 0}$ & $\mathbf{- 1 . 6 9}$ \\
\hline
\end{tabular}

Table 2. Top 50 age-related phosphorylation changes in mouse hearts. Bold indicates unadjusted P-

477 value $<0.05$. Ordered by lowest P-value in Young vs Old Control comparison. Full results can be found in

478 Appendix 2.

\begin{tabular}{|l|c|c|c|}
\hline \multirow{2}{*}{ Peptide (@ indicates site of phosphorylation) } & $\begin{array}{c}\text { Maps to Protein } \\
\text { Phosphorylation Site(s) }\end{array}$ & \multicolumn{2}{|c|}{$\begin{array}{c}\text { Fold Change Relative } \\
\text { to Old Control }\end{array}$} \\
\cline { 2 - 4 } & & Young & Old SS-31 \\
\hline DALLGSNPNKPS@PPSSPSSR & Myom1_S863 & $\mathbf{3 . 5 3}$ & -1.12 \\
\hline ILNPAAIPEGQFIDS@R & Myh6_S740 & $\mathbf{1 . 2 9}$ & 1.41 \\
\hline SNLKPDVEHCTGAGTGS@PLEEEVR & Rbpms_S18 & $\mathbf{- 1 . 7 7}$ & -1.06 \\
\hline GAPVPSTGAPPS@PK & Naca_S765 & $\mathbf{- 2 . 0 0}$ & 1.04 \\
\hline GIS@LEEGALPDVSATR & Xirp1_S295 & $\mathbf{2 . 0 9}$ & 1.06 \\
\hline GAPNALAESPAS@PK & Naca_S1285 & $\mathbf{- 1 . 9 6}$ & 1.01 \\
\hline KAS@LKDSGEYTCETEASK & Obscn_S3321 & $\mathbf{1 . 9 4}$ & 1.24 \\
\hline TVS@QESLTPGKLEINFEQLLR & Nexn_S296 & $\mathbf{- 2 . 0 7}$ & -1.53 \\
\hline IYSHS@GEDIEFGCK & Cfh_S1198 & $\mathbf{2 . 8 5}$ & 1.40 \\
\hline VMHTQCHSTPDS@AEDVRK & Ahsg_S138 & $\mathbf{1 . 8 6}$ & 1.02 \\
\hline SGS@PGPLHSVSGYK & Rbm20_S728 & $\mathbf{- 1 . 3 7}$ & -1.26 \\
\hline VDNARVSSPEVGSADVASIAQK & Fam21_S747 & $\mathbf{1 . 3 4}$ & -1.07 \\
\hline LLTRT@PDIAQR & Stk39_T366 & $\mathbf{- 2 . 2 1}$ & -1.36 \\
\hline GDS@ETDLEALFNAVMNPK & Yap1_S46 & $\mathbf{1 . 7 9}$ & -1.06 \\
\hline TYS@LGSALRPSTSR & Vim_S39 & $\mathbf{3 . 4 0}$ & 1.35 \\
\hline GRS@SLDLDKTPAFLHVK & Nebl_S352 & $\mathbf{1 . 8 9}$ & 1.06 \\
\hline RES@DGTPGGLASLENER & Sorbs1_S432(0:3) & $\mathbf{1 . 9 6}$ & 1.19 \\
\hline LANISVPASES@PR & Tnks1bp1_S300 & $\mathbf{3 . 1 9}$ & $\mathbf{5 . 0 8}$ \\
\hline SRNS@PLLDR & Mark2_S483 & $\mathbf{- 1 . 7 9}$ & -1.33 \\
\hline IYHLPDAES@DEDEDFKEQTR & Sept2_S218 & $\mathbf{1 . 4 1}$ & 1.22 \\
\hline KLVIIES@DLERAEER & Tpm1_S174 & $\mathbf{- 9 . 9 9}$ & - \\
\hline
\end{tabular}




\begin{tabular}{|c|c|c|c|}
\hline SSHCDS@PPRSQTPQDTNR & Bnip3_S60 & -2.33 & -1.34 \\
\hline APS@WIDTGLSEMR & Cryab_S59 & 3.45 & 1.64 \\
\hline AIVSPFHS@PPSTPSSPGIR & Rcsd1_S120 & 1.77 & 1.07 \\
\hline VAKPKS@PEPEATLTFPFLDK & Limch1_S719 & -1.37 & 1.11 \\
\hline AAVGVTGNDITTPPNKEPPPS@PEKK & Map4_S667 & -1.50 & 1.01 \\
\hline NKPRPPSLSLLGGHLPSTLSDGPSS@PR & Trip10_S351 & -1.89 & 1.18 \\
\hline APHSPESPGHSDNPQSSPDSLEAS@PRNPGR & Perm1_S176 & -3.26 & 1.03 \\
\hline VVANS@PANADYQER & Ldb3_S112 (isoform 6) & -1.60 & -1.05 \\
\hline HQCTSGPIVTLQGNDKSTS@PDPDWSSQLER & Synpo2_S220 & -1.72 & -1.08 \\
\hline GCVAS@PVEGGR & Synpo2_S300 & -1.45 & -1.07 \\
\hline ILGSAS@PEEEQEKPILDRPTR & Szrd1_S107 & 1.72 & 1.11 \\
\hline AS@GVTVNDEVIK & CfI2_S3 & -1.51 & 1.05 \\
\hline RGS@LELGNPSAAHLGDELKEVSTAK & Svil_S960 & 1.36 & 1.39 \\
\hline GLGCSDWKPEAGLS@PPR & Tcea3_S113 & -1.48 & 1.20 \\
\hline SKPAAADS@EGEEEEEDTAKEKEPPQQGK & Abcf1_S194 & 1.99 & -1.14 \\
\hline TTS@FAESCKPVQQPSAFGSMK & Gsk3b_s9 & 4.32 & 1.04 \\
\hline MGQAGS@TISNSHAQPFDFPDDSQNAKK & Gja1_S325 & -3.24 & -2.23 \\
\hline VKS@PETVKS@PK & $T t n \_S 34464, S 34470$ & -1.55 & -1.01 \\
\hline VQLLHSQNTS@LINQKK & Myh6_S1724 & 1.27 & 1.21 \\
\hline KVS@KQEEASGGPLAPK & Vasp_S235 & 1.92 & 1.66 \\
\hline GPVS@PPAR & Naca_S257 & -1.37 & 1.14 \\
\hline KRHS@GDFGADAQGAMSK & Mb_S121 & 1.57 & 1.38 \\
\hline SSS@PVTELTAR & Srrm2_S1068 & 1.36 & 1.07 \\
\hline RS@SDPALTGLSTSVSDNNFSSEEPSR & Pard3_S143 & 1.29 & 1.11 \\
\hline VQLLHS@QNTSLINQK & Myh6_S1720 & 1.28 & 1.51 \\
\hline ATEEPS@GTGS@DELIKSDQVNGVLVLSLLDK & Ptrf_S38,S42 & -3.39 & -3.53 \\
\hline EQTAS@APATPLVSK & Cobll1_S300 & -1.47 & 1.17 \\
\hline LLQGLLGS@DDEEQEDPKDYCK & Srpk3_S49 & -1.54 & -1.12 \\
\hline SPS@TIDVVR & Xirp1_S533 & 3.82 & 1.34 \\
\hline
\end{tabular}

480 Table 3. Top 50 age-related protein abundance changes in mouse hearts. Bold indicates unadjusted P-

481 value<0.05. Ordered by lowest P-value in Young vs Old Control comparison. Full results can be found in 482 Appendix 3.

\begin{tabular}{|c|c|c|}
\hline \multirow{2}{*}{ Protein } & \multicolumn{2}{|c|}{$\begin{array}{c}\text { Fold Change Relative } \\
\text { to Old Control }\end{array}$} \\
\cline { 2 - 3 } & Young & Old SS-31 \\
\hline Apoe & $\mathbf{5 . 5 7}$ & -1.30 \\
\hline$C p$ & $\mathbf{7 . 1 2}$ & -1.20 \\
\hline Fetub & $\mathbf{4 . 9 1}$ & -1.51 \\
\hline$H p x$ & $\mathbf{5 . 2 0}$ & -1.25 \\
\hline$T f$ & $\mathbf{1 . 8 9}$ & 1.04 \\
\hline Itih2 & $\mathbf{3 . 7 9}$ & -1.38 \\
\hline Ctsb & $\mathbf{2 . 0 4}$ & -1.08 \\
\hline Lcp1 & $\mathbf{7 . 1 3}$ & -1.84 \\
\hline
\end{tabular}




\begin{tabular}{|c|c|c|}
\hline Sod2 & -1.44 & 1.25 \\
\hline Ahsg & 1.63 & 1.13 \\
\hline$C f l 1$ & 1.82 & -1.23 \\
\hline Ank3 & -6.30 & 1.43 \\
\hline Ppt1 & 6.11 & 1.25 \\
\hline Vtn & 7.26 & -1.46 \\
\hline$M t 1$ & 2.02 & -1.30 \\
\hline Calu & 2.04 & -1.17 \\
\hline S100a11 & 2.69 & -1.78 \\
\hline Hspg2 & 1.38 & -1.25 \\
\hline Kng1 & 2.46 & -1.06 \\
\hline Asph & -1.56 & 1.33 \\
\hline Kng1 & 2.40 & -1.02 \\
\hline $\operatorname{Tgm} 2$ & 1.51 & -1.05 \\
\hline Minos1 & -2.73 & -1.14 \\
\hline$T g f b i$ & 5.33 & -1.21 \\
\hline Itih4 & 15.51 & -1.19 \\
\hline Endog & -1.63 & 1.06 \\
\hline Mt2 & 3.44 & -1.31 \\
\hline Dusp3 & 1.39 & -1.15 \\
\hline Anxa5 & 1.56 & 1.05 \\
\hline$W f s 1$ & 6.33 & -1.33 \\
\hline Fabp4 & 1.27 & -1.20 \\
\hline$X d h$ & 2.20 & -1.21 \\
\hline Itih1 & 3.20 & 1.05 \\
\hline Lama2 & -1.30 & 1.04 \\
\hline Cap1 & 2.20 & 1.10 \\
\hline Bgn & 2.73 & -1.08 \\
\hline Impdh2 & 2.49 & -1.62 \\
\hline Cfh & 4.65 & 1.06 \\
\hline$N t 5 c 3$ & 1.47 & -2.14 \\
\hline Abat & 7.83 & -1.30 \\
\hline Cltc & 1.47 & -1.13 \\
\hline Art3 & -1.63 & 1.36 \\
\hline Rps2 & 1.42 & -1.09 \\
\hline Picalm & 3.58 & -1.26 \\
\hline Acot7 & 1.44 & -1.62 \\
\hline$G c$ & 2.13 & 1.08 \\
\hline Tppp & -1.78 & 1.76 \\
\hline Ctsd & 1.54 & 1.17 \\
\hline Pex3 & 1.50 & 1.00 \\
\hline Bdh1 & 1.63 & -1.22 \\
\hline
\end{tabular}




\begin{tabular}{|c|c|c|c|c|}
\hline $\begin{array}{c}\text { Peptide (@ indicates site of } \\
\text { phosphorylation) }\end{array}$ & $\begin{array}{c}\text { Maps to Protein } \\
\text { (site indicated } \\
\text { when } \\
\text { phosphorylated) }\end{array}$ & Young & $\begin{array}{c}\text { Old } \\
\text { Control }\end{array}$ & $\begin{array}{l}\text { Old SS- } \\
31\end{array}$ \\
\hline EQANLFSEWLR & Ttn & $1.30 \mathrm{E}-04$ & $1.52 \mathrm{E}-04$ & $1.42 \mathrm{E}-04$ \\
\hline EQANLFS@EWLR & Ttn_S4080 & $1.08 \mathrm{E}-05$ & $1.45 \mathrm{E}-05$ & $1.59 \mathrm{E}-05$ \\
\hline VIQSYSIR & Actn2 & $1.03 \mathrm{E}-03$ & $1.12 \mathrm{E}-03$ & $1.10 \mathrm{E}-03$ \\
\hline VIQSYS@IR & Actn2_S590 & 2.53E-05 & $1.83 \mathrm{E}-05$ & $1.81 E-05$ \\
\hline RGTGGVDTAAVGAVFDISNADR & $C k m$ & 8.55E-05 & $8.81 \mathrm{E}-05$ & 8.63E-05 \\
\hline RGT@GGVDTAAVGAVFDISNADR & Ckm_T322 & $1.45 \mathrm{E}-05$ & $1.56 \mathrm{E}-05$ & $1.76 \mathrm{E}-05$ \\
\hline RASAPLPGFSAPGR & Hspb6 & $9.43 \mathrm{E}-06$ & $1.29 \mathrm{E}-05$ & $1.34 \mathrm{E}-05$ \\
\hline RAS@APLPGFSAPGR & Hspb6_S16 & $6.95 \mathrm{E}-05$ & $5.03 \mathrm{E}-05$ & $6.25 \mathrm{E}-05$ \\
\hline RDSKLEAPAEEDVWEILR & $c M y B P-C$ & $1.56 \mathrm{E}-06$ & $2.77 \mathrm{E}-06$ & 2.61E-06 \\
\hline RDS@KLEAPAEEDVWEILR & cMyBP-C_S307 & 2.99E-05 & $2.90 \mathrm{E}-05$ & $2.54 \mathrm{E}-05$ \\
\hline SSSRAEANDQDAIQEK & Myot & 2.87E-08 & $4.37 \mathrm{E}-07$ & $5.80 \mathrm{E}-07$ \\
\hline SSS@RAEANDQDAIQEK & Myot_S231 & $3.54 \mathrm{E}-07$ & $6.84 \mathrm{E}-07$ & $6.72 \mathrm{E}-07$ \\
\hline RASTIEMPQQAR & $P / n$ & $5.66 \mathrm{E}-06$ & $6.85 \mathrm{E}-06$ & $7.16 \mathrm{E}-06$ \\
\hline RAS@T@IEMPQQAR & PIn_S15,T16 & $2.23 E-04$ & 2.09E-04 & $2.32 \mathrm{E}-04$ \\
\hline
\end{tabular}
glutathionylation analysis. Each dot represents one detected peptide. The horizontal dotted line indicates $F D R=0.01$. The vertical dotted lines represent $\log 2$ ratios $>1$ and $<-1$. (C) Histogram of \%occupancy of cysteine residues on detected peptides. (D) P-value heatmap of protein S-glutathionylation changes in canonical pathways based on all changes with an unadjusted $\mathrm{P}<0.05$. Top 25 pathways, ranked by $\mathrm{P}$-value, are shown. (E) Row-normalized $z$-score heatmaps of significant $(P<0.05) S$-glutathionylation differences in selected pathways. (F) Mean GSH bound to protein by treatment group normalized to Control, as determined by HPLC. $\mathrm{N}=5$ for all groups.

Figure 2. Analysis of Phosphorylation Changes in Mouse Hearts. (A-B) Volcano plots of protein global phosphorylation analysis. Each dot represents one detected peptide. The horizontal dotted lines indicate unadjusted $\mathrm{P}<0.01$ and $\mathrm{P}<0.05$ as noted on the chart. The vertical dotted lines represent $\log 2$ ratios $>1$ and $<-1$. (C) P-value heatmap of protein phosphorylation changes in canonical pathways based on all

501 changes with an unadjusted $\mathrm{P}<0.05$. Top 25 pathways, ranked by P-value, are shown. (D) Row-normalized z-score heatmaps of significant $(P<0.05)$ S-phosphorylation differences in selected pathways. 
504 Figure 3. Analysis of Protein Abundance Changes in Mouse Hearts. (A-B) Volcano plots of protein

505 abundance analysis. Each dot represents one protein. The horizontal line indicates FDR<0.01. (C) P-value

506 heatmap of protein abundance changes in canonical pathways based on all changes with an unadjusted

$507 \mathrm{P}<0.05$. Top 25 pathways, ranked by $\mathrm{P}$-value, are shown. (D) Row-normalized $z$-score heatmaps of 508 significant $(\mathrm{P}<0.05)$ protein abundance differences in selected pathways.

509

510 Figure 4. Parallel Reaction Monitoring Mass Spectroscopy of Targeted Phosphorylation Sites in Mouse

511 Hearts. Sites targeted for analysis were (A-B) Ttn_S4080, (C-D) Actn2_S590, (E-F) cMyBP-C_S307, (G-H)

512 Myot_S231, (I-J) Pln_S15,T16, (K-L) Hspb6_S16, (M-N) Ckm_T322. Both the phosphorylated and 513 unphosphorylated form of the associated peptide was analyzed for each. Peptide sequences are provided 514 in Table 3. Young $N=5$, Old Control $N=21$, Old SS-31 $N=14$. 
bioRxiv preprint doi: https://doi.org/10.1101/2021.08.06.455402; this version posted August 9, 2021. The copyright holder for this preprint (which was not certified by peer review) is the author/funder. All rights reserved. No reuse allowed without permission.

Page 23

A

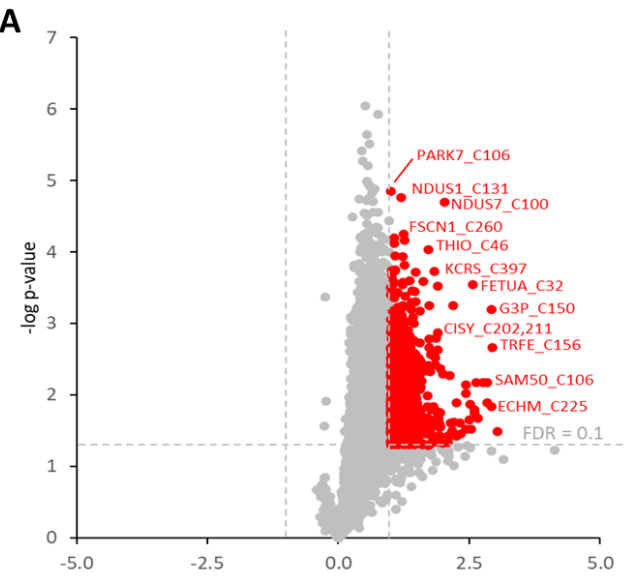

C

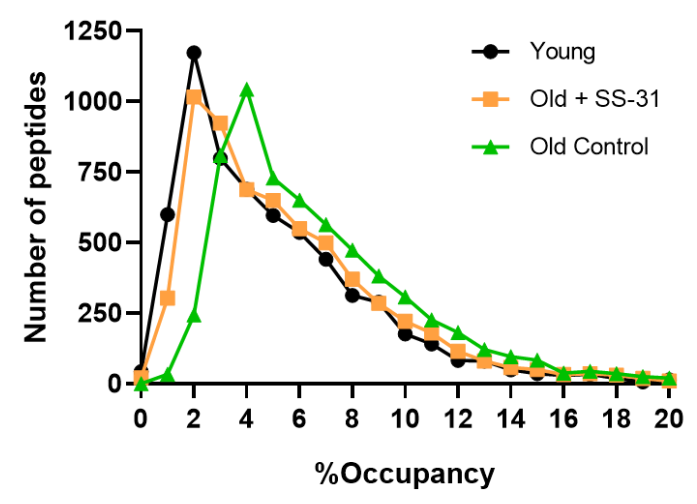

E

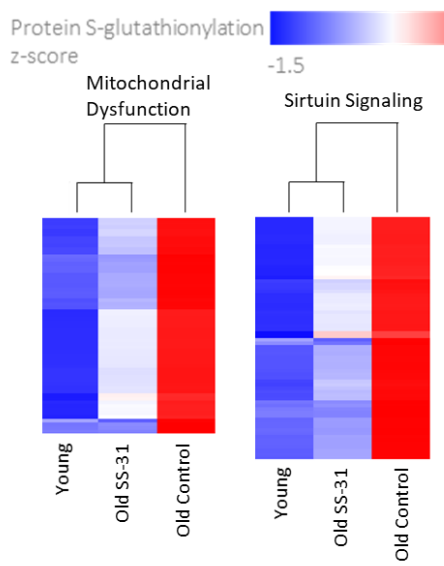

F

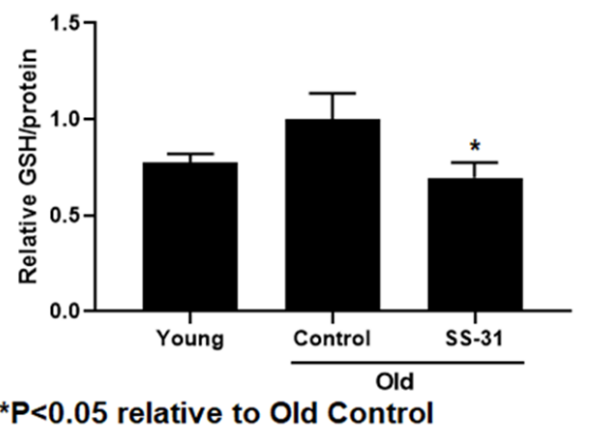

B

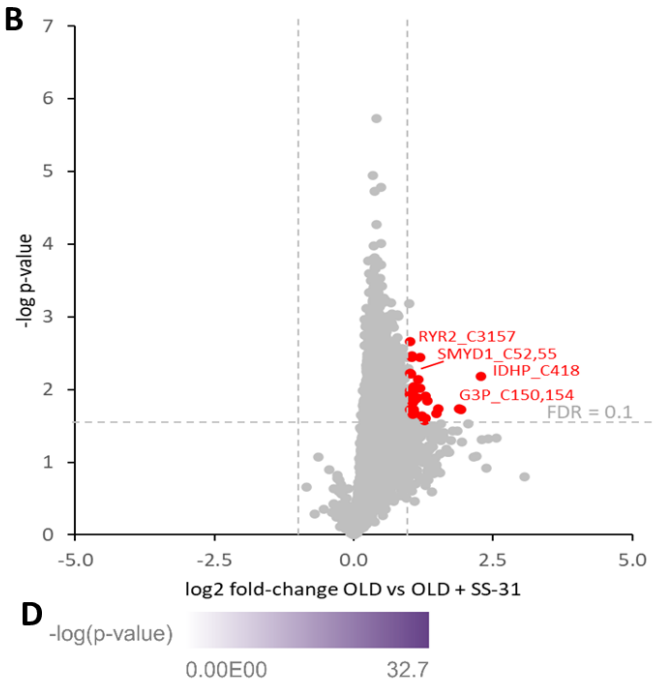

Mitochondrial Dysfunction Sirtuin Signaling Pathway Oxidative Phosphorylation Actin Cytoskeleton Signaling Valine Degradation I EIF2 Signaling Protein Ubiquitination Pathway Epithelial Adherens Junction Signaling ILK Signaling TCA Cycle II (Eukaryotic) Integrin Signaling Remodeling of Epithelial Adherens Junctions Fatty Acid $\beta$-oxidation I Germ Cell-Sertoli Cell Junction Signaling Glycolysis I

Regulation of Actin-based Motility by Rho Regulation of elF4 and p70S6K Signaling Gluconeogenesis I

NRF2-mediated Oxidative Stress Response Isoleucine Degradation I

Protein Kinase A Signaling

Breast Cancer Regulation by Stathmin 1 Calcium Signaling Sertoli Cell-Sertoli Cell Junction Signaling Apelin Adipocyte Signaling Pathway 
A

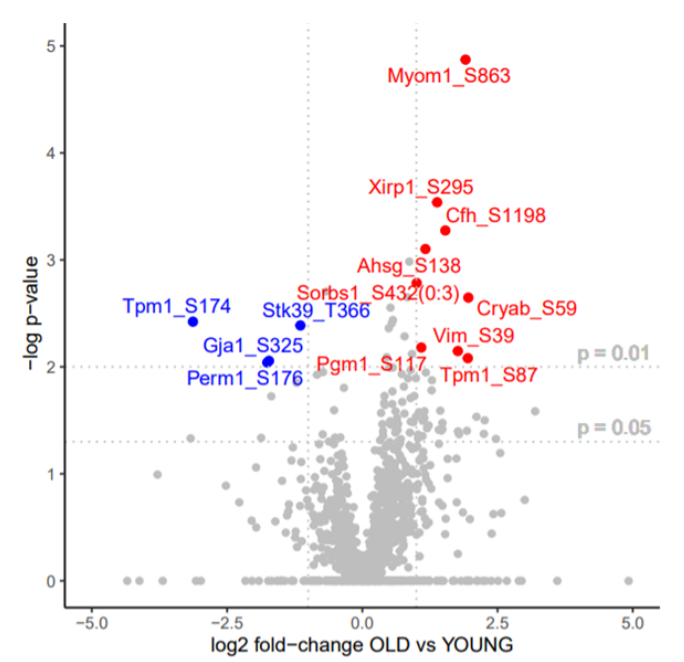

C $-\log (p-v a l u e)$

$$
0.00 \mathrm{E} 00
$$$$
4.1
$$

ILK Signaling

RhoA Signaling

Integrin Signaling

Actin Cytoskeleton Signaling

HIPPO signaling

Caveolar-mediated Endocytosis Signaling

Epithelial Adherens Junction Signaling

Cdc42 Signaling

Protein Kinase A Signaling

Signaling by Rho Family GTPases

14-3-3-mediated Signaling

Adrenomedullin signaling pathway

Tight Junction Signaling

Branched-chain $\alpha$-keto acid Dehydrogenase Complex

Wnt $/ \beta$-catenin Signaling

Insulin Receptor Signaling

Cell Cycle: G1/S Checkpoint Regulation

Axonal Guidance Signaling

Cyclins and Cell Cycle Regulation

HER-2 Signaling in Breast Cancer

GDP-glucose Biosynthesis

Glucose and Glucose-1-phosphate Degradation

Sucrose Degradation V (Mammalian)

PD-1, PD-L1 cancer immunotherapy pathway

Rac Signaling

\section{Figure 2. Analysis of Phosphorylation Changes in Mouse Hearts}

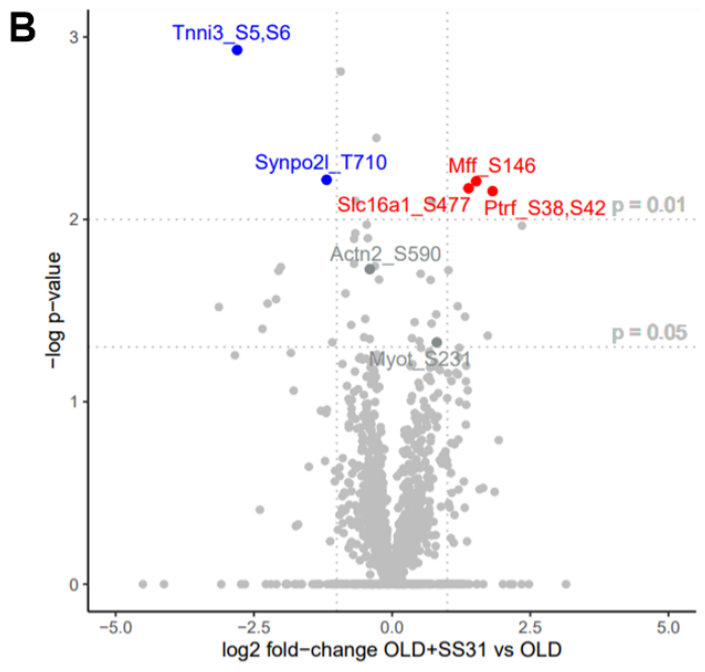

D Phospho peptide
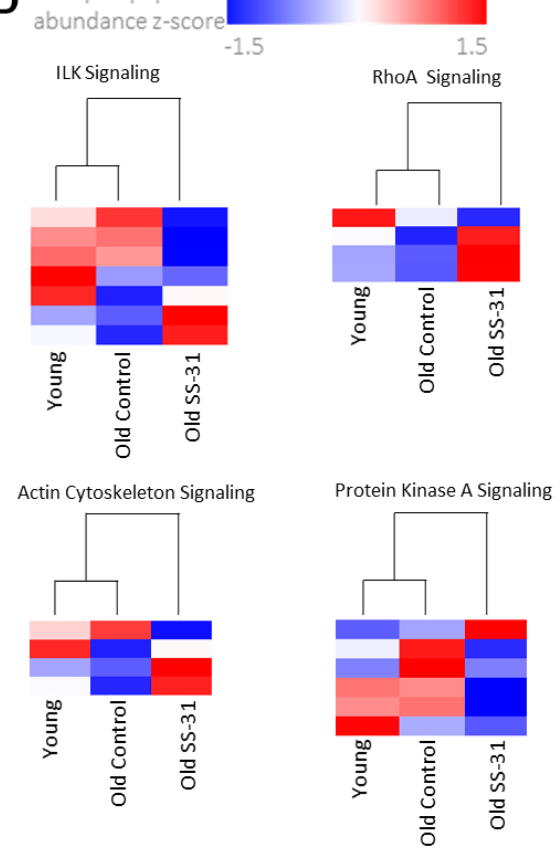
bioRxiv preprint doi: https://doi.org/10.1101/2021.08.06.455402; this version posted August 9, 2021. The copyright holder for this preprint (which was not certified by peer review) is the author/funder. All rights reserved. No reuse allowed without permission.

A

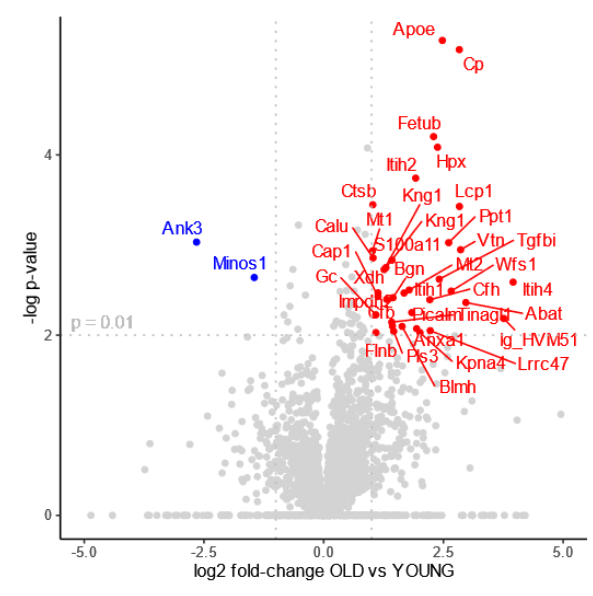

C $\log (\mathrm{p}$-value $)$

$$
0.00 \mathrm{E} 00
$$

Phagosome Maturation

Germ Cell-Sertoli Cell Junction Signaling Remodeling of Epithelial Adherens Junctions Epithelial Adherens Junction Signaling Coronavirus Replication Pathway Acute Phase Response Signaling Actin Cytoskeleton Signaling Clathrin-mediated Endocytosis Signaling Sertoli Cell-Sertoli Cell Junction Signaling 14-3-3-mediated Signaling FXR/RXR Activation Purine Nucleotides Degradation II (Aerobic) Axonal Guidance Signaling LXR/RXR Activation

Guanosine Nucleotides Degradation III Urate Biosynthesis/Inosine 5'-phosphate Degradation Regulation of Actin-based Motility by Rho Integrin Signaling Signaling by Rho Family GTPases Huntington's Disease Signaling Mitochondrial Dysfunction Gap Junction Signaling Semaphorin Signaling in Neurons RhoGDI Signaling RhoA Signaling
B

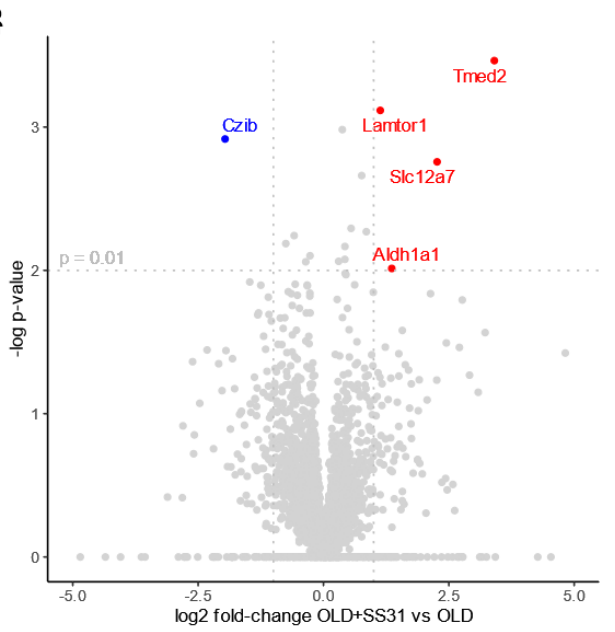

D Protein abundance z-score Actin Cytoskeleton Signaling
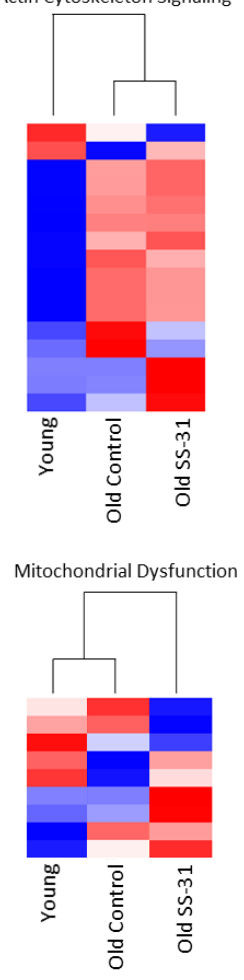
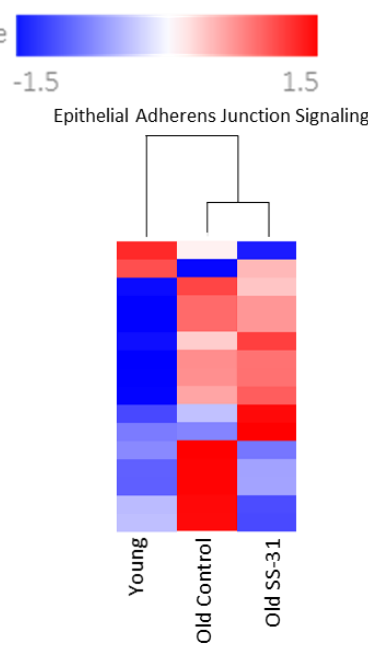

RhoA Signaling

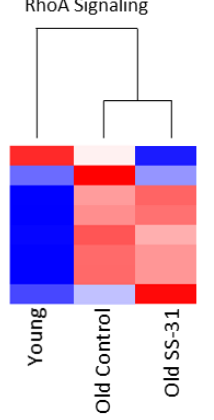


$\mathbf{A}_{\text {Ttn } \$ 4080 \text { Unphosphorylated }}$

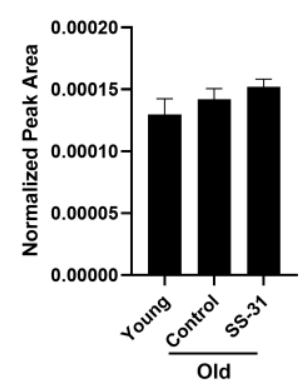

$E_{\text {Mybpc3 S307 Unphosphorylated }}$

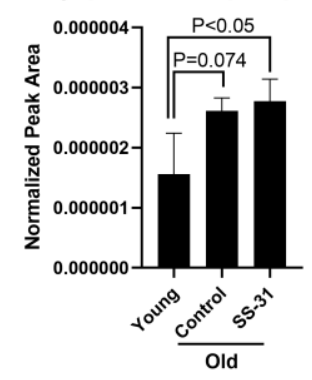

I PIn S15,T16 Unphosphorylated

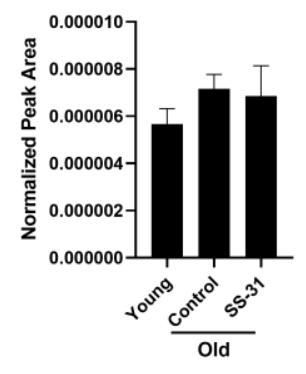

M ckm T322 Unphosphorylated

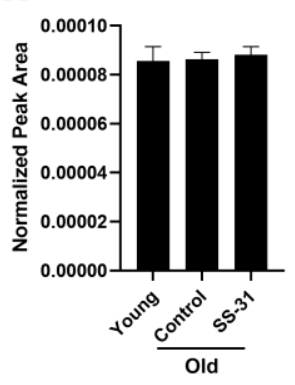

B

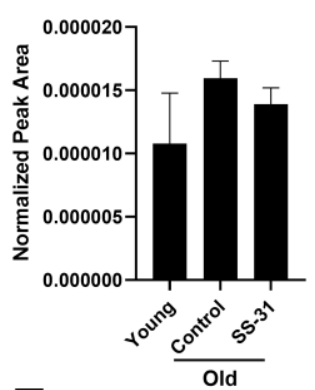

$\boldsymbol{F}_{\text {Mybpc3 S307 Phosphorylated }}$

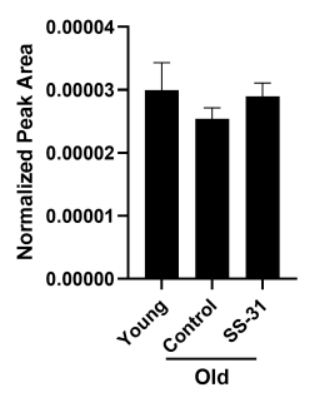

J Pln S15,T16 Phosphorylated

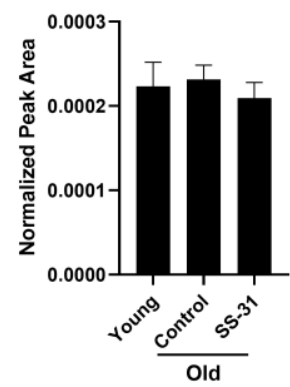

N Ckm T322 Phosphorylated

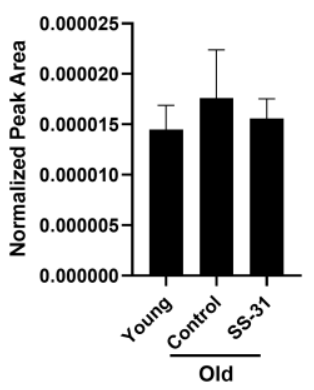

C Actn2 S590 Unphosphorylated

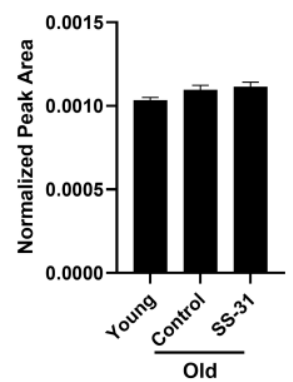

G

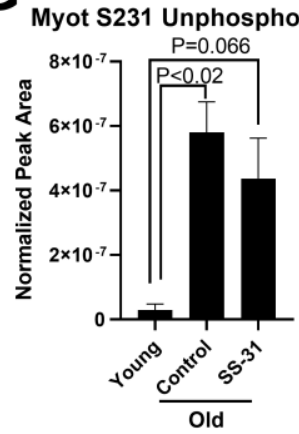

K Hspb6 S16 Unphosphorylated

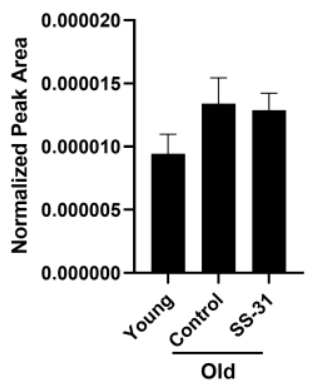

Actn2 S590 Phosphorylated

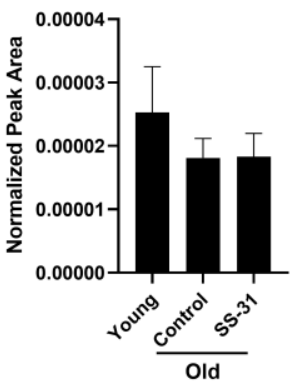

H

Myot S231 Phosphorylated

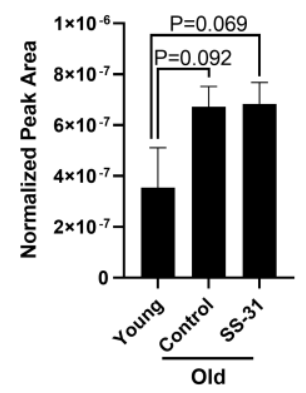

L Hspb6 S16 Phosphorylated

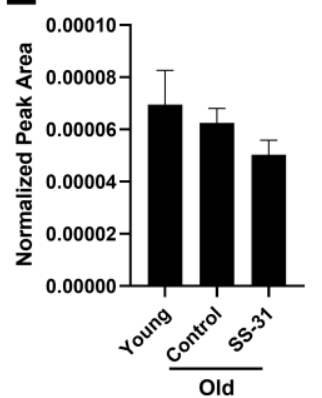

